\title{
A Study toward Disintegration of Coronavirus using Near-infrared Light Therapy
}

\author{
Pradeep Kumar Pasricha \\ Scientist-F (Retired) \\ National Physical Laboratory \\ New Delhi, India
}

\begin{abstract}
A coronavirus is a protein molecule covered by a thin layer of fat. It binds to the human cells and replicates within the cells. The near-infrared light produces intense heat, and also penetrates tissue in order to deliver energy to cells. In the present study, an ideal model of a cell full of viruses is considered. The propagation of near-infrared electromagnetic waves in such a dielectric medium is examined to check whether the near-infrared wavelengths may deliver energy to the medium. With the known observations of refractive indices of cell and virus, below the near-infrared wavelengths, it is computed that the near-infrared wavelengths deliver "excessive" energy to the dielectric medium. Thus, light at near-infrared wavelengths may penetrate and heat tissue affected by coronavirus. It may result in the melting of layer of fat of coronavirus in cells. Near-infrared light therapy at discrete wavelengths in the band $(700-900$ $\mathrm{nm}$ ) may reduce severity of conditions in the gravely affected coronavirus patients.
\end{abstract}

\section{Introduction}

The model calculations presented in this report are in view of a pdf file mailed by Johns Hopkins University, which has quoted reference [1]. Accordingly, the corona virus is a protein molecule covered by a thin layer of fat, the lipid membrane. The virus binds to the host cells and then penetrates to release the viral DNA, which controls the cell's metabolism. It then replicates itself within the cells of its host and forms new viruses. It is the thin layer of fat that protects the virus from being destroyed. However, this thin layer of fat may be dissolved by heat. Now, it is known that near-infrared light at wavelengths between $700-1400 \mathrm{~nm}$ generates the "most intense" heat in the infrared spectrum $700-2500 \mathrm{~nm}$. The wavelengths between $700-1400 \mathrm{~nm}$ form the near-infrared wavelength band. These wavelengths penetrate into human tissue. These are absorbed, delivering energy to cells. The near-infrared wavelengths heat tissue for the consequent health-related benefits [2]. The corresponding therapy is called near-infrared light therapy. The longer wavelengths in the band are also known to penetrate "deep" into tissue, compared to the shorter wavelengths, in the near-infrared band [2].

In the present study, an ideal model of a cell-plus-viruses has been envisaged, where a large number of viruses are ideally placed in a cell so as to form an ideal spherical microcavity at near-infrared wavelengths. The dielectric medium of such an ideal microcavity has been 
examined to determine whether such a dielectric medium may have "excessive" (photon) energy tranfer, compared to "normal" energy transfer, by near-infrared wavelengths.

\section{Observations}

Basic Data on Virus

Size of virus: $20-400 \mathrm{~nm}$ (nanometer, 1 nanometer $=10^{-9}$ meters) [3]

Diameter of virus, assumed spherical, in the present model:

(i) $100 \mathrm{~nm}$, lower portion of near-infrared band

(ii) $200 \mathrm{~nm}$, near-infrared band

Radius of virus in the present model:

(i) $50 \mathrm{~nm}$, lower portion of near-infrared band

(ii) $100 \mathrm{~nm}$, near-infrared band

Refractive index of virus at $532 \mathrm{~nm}: 1.45$ [4]

Refractive index of virus in the near infrared band $700-1400 \mathrm{~nm}$, in the present model: 1.45

\section{$\underline{\text { Basic Data on Human Cell }}$}

Size of cell (nucleus + cytoplasm): $8000 \mathrm{~nm}$

Thickness of cell membrane: $10 \mathrm{~nm}$

Thickness of membrane, assumed spherical, in the present model:

$10 \mathrm{~nm}$

Thickness of cell, assumed spherical, in the present model:

$8000 \mathrm{~nm}$

Refractive index of cell: 1.36 - 1.38 [5]

Refractive index of cell at $510-750 \mathrm{~nm}: 1.33-1.38$ [6]

Refractive index of cell in the near infrared band $700-1400 \mathrm{~nm}$, assumed in the present model:

(i) 1.37, lower portion of near-infrared band

(ii) 1.30 , near-infrared band

(iii) 1.31 , near-infrared band

(iv) 1.32 , near-infrared band

(v) 1.37, near-infrared band 


\section{Methodology}

(i) Radius of virus $r=50 \mathrm{~nm}$, cell refractive index $n=1.37$, lower portion of near-infrared band

The design of an ideal spherical microcavity at $650 \mathrm{~nm}$ of viruses of radius $\mathrm{r}=50 \mathrm{~nm}$ (diameter $100 \mathrm{~nm}$ ) and refractive index $n=1.45$ in a cell of refractive index $n=1.37$ is presented in Figure 1.

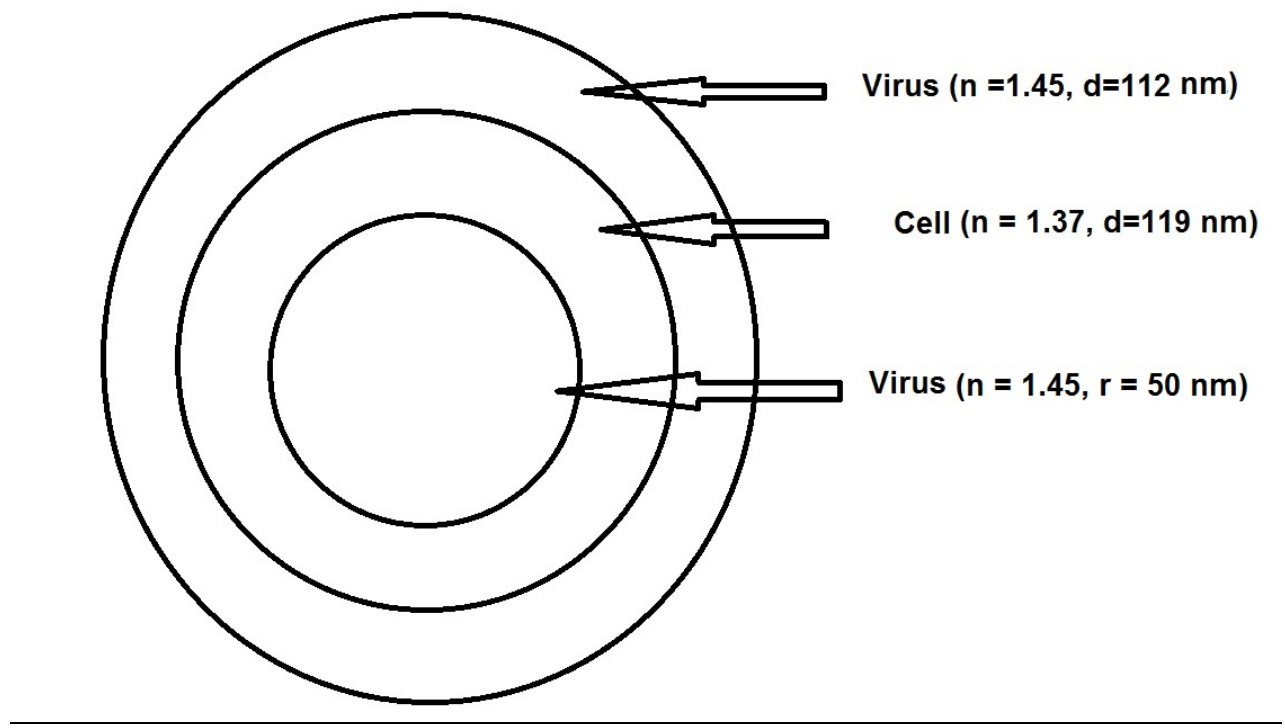

Figure 1. A spherical microcavity consisting of virus-core of radius $\mathrm{r}=50 \mathrm{~nm}(\mathrm{n}=1.45)$, and eight pairs of layers of cell $(n=1.37)$ and virus $(n=1.45)$. Two pairs of layers of virus and cell are depicted. There are 17 viruses in a cell, overall.

Such a microcavity is termed as Bragg Onion Resonator without the stem [7]. (The stem is an opening, which is used to excite TE/TM modes of monochromatic electromagnetic waves.) It is also termed as Bragg Reflector [8]. The formulation of a Bragg Onion Resonator for $\mathrm{TE}_{10}$ (and $\mathrm{TM}_{10}$ mode) of electromagnetic wave propagation has been coded in the $\mathrm{C}$ programming language [9]. This $\mathrm{C}$ programming code has been used to compute the wavelength of resonance in the spherical microcavity in Figure 1. (A similar set of computations can be made for $\mathrm{TM}_{10}$ mode.)

The thickness of virus-layer is $d=650 / 4 * 1.45 \approx 112 \mathrm{~nm}$, which approximately equals the size of virus-core (diameter) of $100 \mathrm{~nm}$. The thickness of cell-layer is $\mathrm{d}=650 / 4 * 1.37 \approx 119 \mathrm{~nm}$. There are 17 viruses in a cell. The total thickness of the spherical microcavity, including the thickness of the membrane, is roughly $4000 \mathrm{~nm}$; the cell size (diameter) is $8000 \mathrm{~nm}$.

The spherical microcavity in Figure 1 implies resonance at wavelength $694.1 \mathrm{~nm}$. The quality of occurrence of resonance in the microcavity is "good". A resonance in a cavity implies "excessive" transfer of (photon) energy at the computed wavelength. (In the present study, it suffices to qualify the meeting of the condition of occurrence of resonance in a microcavity as "high" or "good" or "fair".) 
It may be noted that one cannot design an ideal spherical microcavity at $650 \mathrm{~nm}$ for the virus-core of radius $r=100 \mathrm{~nm}$ (diameter $200 \mathrm{~nm}$ ), since the virus-core size $(200 \mathrm{~nm})$ is not roughly equal to the designed virus-layer thickness $\mathrm{d} \approx 112 \mathrm{~nm}$ at $650 \mathrm{~nm}$.

\section{(ii) Radius of virus $r=100 \mathrm{~nm}$, cell refractive index $n=1.30$, in near-infrared band}

The design of an ideal spherical microcavity of viruses of radius $r=100 \mathrm{~nm}$ (diameter 200 $\mathrm{nm}$ ) and refractive index $\mathrm{n}=1.45$ in a cell of refractive index $\mathrm{n}=1.30$ at $1050 \mathrm{~nm}$ is considered.

The thickness of virus-layer is $\mathrm{d}=1050 / 4 * 1.45 \approx 181 \mathrm{~nm}$, which approximately equals the size of virus-core (diameter) of $200 \mathrm{~nm}$. The thickness of cell-layer is $d=1050 / 4 * 1.30 \approx 202$ $\mathrm{nm}$. There are 10 viruses in a cell. The total thickness of the spherical microcavity, including the thickness of the membrane, is roughly $4000 \mathrm{~nm}$; the cell size (diameter) is $8000 \mathrm{~nm}$.

The spherical microcavity implies "good" resonance at wavelength $904.8 \mathrm{~nm}$. A resonance in a cavity implies "excessive" tranfer of (photon) energy at the computed wavelength. (In the present study, it suffices to qualify the meeting of the condition of the occurrence of resonance in a microcavity as "high" or "good" or "fair".)

It may be noted that one cannot design an ideal spherical microcavity at $1050 \mathrm{~nm}$ for the virus-core of radius $r=50 \mathrm{~nm}$ (diameter $100 \mathrm{~nm}$ ), since the virus-core size $(100 \mathrm{~nm})$ is not roughly equal to the designed virus-layer thickness $\mathrm{d} \approx 181 \mathrm{~nm}$ at $1050 \mathrm{~nm}$.

(iii) Radius of virus $r=100 \mathrm{~nm}$, cell refractive index $\mathrm{n}=1.31$, in near-infrared band

The design of an ideal spherical microcavity of viruses of radius $r=100 \mathrm{~nm}$ (diameter 200 $\mathrm{nm}$ ) and refractive index $\mathrm{n}=1.45$ in a cell of refractive index $\mathrm{n}=1.31$ at $1050 \mathrm{~nm}$ is considered.

The thickness of virus-layer is $d=1050 / 4 * 1.45 \approx 181 \mathrm{~nm}$, which approximately equals the size of virus-core (diameter) of $200 \mathrm{~nm}$. The thickness of cell-layer is $d=1050 / 4 * 1.31 \approx 200$ $\mathrm{nm}$. There are 10 viruses in a cell. The total thickness of the spherical microcavity, including the thickness of the membrane, is roughly $4000 \mathrm{~nm}$; the cell size (diameter) is $8000 \mathrm{~nm}$.

The spherical microcavity implies "good" resonance at wavelength $902.8 \mathrm{~nm}$. A resonance in a cavity implies "excessive" tranfer of (photon) energy at the computed wavelength. (In the present study, it suffices to qualify the meeting of the condition of the occurrence of resonance in a microcavity as "high" or "good" or "fair".)

(iv) Radius of virus $r=100 \mathrm{~nm}$, cell refractive index $n=1.32$, in near-infrared band

The design of an ideal spherical microcavity of viruses of radius $r=100 \mathrm{~nm}$ (diameter 200 $\mathrm{nm}$ ) and refractive index $\mathrm{n}=1.45$ in a cell of refractive index $\mathrm{n}=1.32$ at $1050 \mathrm{~nm}$ is considered.

The thickness of virus-layer is $\mathrm{d}=1050 / 4 * 1.45 \approx 181 \mathrm{~nm}$, which approximately equals the size of virus-core (diameter) of $200 \mathrm{~nm}$. The thickness of cell-layer is $\mathrm{d}=1050 / 4 * 1.32 \approx 200$ $\mathrm{nm}$. There are 10 viruses in a cell. The total thickness of the spherical microcavity, including the thickness of the membrane, is roughly $4000 \mathrm{~nm}$; the cell size (diameter) is $8000 \mathrm{~nm}$.

The spherical microcavity implies "fair" resonance around wavelength $1188 \mathrm{~nm}$. A resonance in a cavity implies "excessive" transfer of (photon) energy at the computed 
wavelength. (In the present study, it suffices to qualify the meeting of the condition of the occurrence of resonance in a microcavity as "high" or "good" or "fair".)

\section{(v) Radius of virus $r=100 \mathrm{~nm}$, cell refractive index $n=1.37$, in near-infrared band}

The design of an ideal spherical microcavity of viruses of radius $r=100 \mathrm{~nm}$ (diameter 200 $\mathrm{nm}$ ) and refractive index $n=1.45$ in a cell of refractive index $n=1.37$ at $1050 \mathrm{~nm}$ is considered.

The thickness of virus-layer is $\mathrm{d}=1050 / 4 * 1.45 \approx 181 \mathrm{~nm}$, which approximately equals the size of virus-core (diameter) of $200 \mathrm{~nm}$. The thickness of cell-layer is $d=1050 / 4 * 1.37 \approx 192$ $\mathrm{nm}$. There are 10 viruses in a cell. The total thickness of the spherical microcavity, including the thickness of the membrane, is roughly $4000 \mathrm{~nm}$; the cell size (diameter) is $8000 \mathrm{~nm}$.

The spherical microcavity implies "fair" resonance around wavelength $1175 \mathrm{~nm}$. A resonance in a cavity implies "excessive" transfer of (photon) energy at the computed wavelength. (In the present study, it suffices to qualify the meeting of the condition of the occurrence of resonance in a microcavity as "high" or "good" or "fair".)

\section{Data Analysis}

In a real-world scenario, the formation of viruses in a cell is a random process. One has to consider propagation of near-infrared electromagnetic waves in a random dielectric medium. The data on the dielectric medium comprising virus and cell need also to be appropriate to nearinfrared wavelengths. The present study is desired in the prevailing dire coronavirus situation. The aim of the study is to adopt the existing dielectric data in an ideal-world scenario, to compute "excessive" transfer of (photon) energy to the dielectric medium of cells-plus-viruses, if it exists, compared to "normal" delivery of energy by near-infrared waves to tissue of cells alone. Such a study may imply "reasonable excessive" delivery of energy by near-infrared waves to tissue of cells-plus-viruses in a real world scenario, which is the need of the hour.

The virus sizes of $100 \mathrm{~nm}$ and 200 are reasonable estimates of small- and large-size virus. The refractive index of 1.45 of virus is also a reasonable value. The refractive of cell of 1.37 is appropriate to lower limit of near-infrared band of $700-1400 \mathrm{~nm}$. In the present study, the refractive index of cell is taken 1.30, 1.31 and 1.32 in the near-infrared band. (The dielectric medium is not an intrinsically absorbing medium of light. Therefore, refractive index decreases with wavelength of light. In an intrinsically absorbing medium, the refractive index increases with wavelength.)

The propagation of near-infrared light in an ideal dielectric medium of cell-plus-viruses implies "excessive" absorption of (photon) energy at the computed wavelength, compared to "normal" absorption by tissue.

The (photon) quantum of energy $(\mathrm{h} v, \mathrm{~J})$ at $1000 \mathrm{~nm}$ wavelength, with the usual notation of symbols, is two-orders of magnitude higher than kinetic unit of energy (kT, J) at $300 \mathrm{~K}$.

\section{Conclusion}

The observations on the refractive index of virus and human cell are known at below the near-infrared wavelength band. The refractive index of cell may be "logically" decreased in the 
near-infrared band. The model calculations in the present study adopt values of refractive index of cell of 1.30, 1.31 and 1.32. The virus of size $\sim 100 \mathrm{~nm}$ and $\sim 200 \mathrm{~nm}$ have been used in the model calculations. The fat layer of virus in cells may melt by absorption, and heating of tissue, of near-infrared light at discrete wavelengths in the band $(700-900 \mathrm{~nm})$.

The near-infrared light therapy may provide relief to patients moderately and severely affected by coronavirus. However, it may not be useful to minor-affected coronavirus patients. The near-infrared device, such as, LED/laser may be suitably utilized. The level (optical power output, in milliwatts/watts), duration (seconds/minutes) and any side effects of near-infrared light therapy can only be provided by experts.

The temperature of melting of lipid membrane (fat layer of virus) for a variety of lipids commences around $24^{\circ} \mathrm{C}$ to $46^{\circ} \mathrm{C}$ [10]. The solar radiation in the visible and infrared spectrum, $(250-2500 \mathrm{~nm})$, heats both earth and atmosphere through a variety of physical processes. At night, the earth reradiates solar radiation in the longer wavelength spectrum around $(750-30000$ $\mathrm{nm})$ to maintain earth's and atmosphere's temperature, again through a variety of physical processes. There is likely to be melting of coronavirus lipid membrane (fat layer), of viruses in the environment, by heating in summer months. It is expected that the rising of temperature in summer months shall lead to "natural sanitization" of the environment by the solar radiation. It may lead to decrease in coronavirus cases in summer months.

\section{Acknowledgement}

The author gratefully acknowledges Dr. B. Madhava Reddy, Dr. Hirday N. Dutta, Dr. Mohan K. Raina and Dr. Arun K. Upadhayaya of the National Physical Laboratory for their helpful comments.

\section{References}

[1] Gallagher et al., "Alteration of the $\mathrm{pH}$ dependence of coronavirus-induced cell fusion: effect of mutations in the spike glycoprotein", Journal of Virology, vol. 65, pages 1916-1928, 1991.

(Google search: article by Gallagher et al., 1991)

[2] https://www.sunlighten.com/blog/difference-light-therapy-near-infrared-therapy/ https://www.ncbi.nlm.nih.gov/pmc/articles/PMC5653719

(Google search: near-infrared light therapy)

[3] abyss.uoregon.edu/js/glossary/virus.html.

(Google search: size and shape of virus. Quoted in: Encyclopedia Britannica)

[4] Virus-NanoFCM, Angew. Chem. Int. Ed., vol. 55, pages 10239-10243, 2016.

(Google search: Virus-NanoFCM. NanoFCM Inc., D6 Thane Road, Nottingham, UK)

[5] (Liu et al., "Cell refractive index for cell biology and disease diagnosis: past, present and future", Lab Chip, vol. 16, pages 634-644, 2016.

(Google search: Lab Chip, vol. 16, pages 634-644, 2016. Royal Society of Chemistry, RSC Publishing, UK) 
[6] Steelman et al., "Is the nuclear refractive index lower than cytoplasm? Validation of phase measurements and implications for light scattering technologies", J. Biophotonics, vol. 10, pages 1714-1722, 2017.

(Google search: refractive index of cell at $540-720 \mathrm{~nm}$ )

[7] $\mathrm{Xu}$ et al., "High-quality-factor Bragg onion resonators with omnidirectional reflector cladding", Opt. Lett., vol. 28, pages 2144-2146, 2003.

[8] Kaliteevski et al., "Optical eigenmodes of a multilayered spherical microcavity", J. Mod. Opt., vol. 48, pages 1503-1516, 2001.

[9] Pasricha, P. K., Numerical, Statistical and Computational Methods for the Physical Sciences With C, two volumes, to be published.

[10] https://www.sciencedirect.com

https://www.accuweather.com/en/weather-news/how-does-cold-weather-impact-the-spreadsurvival-of-the-flu-virus/342182.

(Google search: lipid melting temperature in virus) 